\title{
Calibration of the Ultrasonic Sensor-Range Finder by the Laser Interferometer
}

\author{
Svyatoslav Yatsyshyn, Dr. Sc., Prof.e-mail: slav.yat@gmail.com; Ihor Nazarkevych, Ph.D. \\ student; \\ Lviv Polytechnic National University, Ukraine \\ Rostyslav Mastylo, Ph.D., \\ Technical University-Ilmenau, Germany
}

\section{Abstract}

The accuracy characteristics of the ultrasonic range finder are studied in the work. The latter has been calibrated by a laser interferometer. The problems that arise are described. The calibration method was implemented with the help of a measuring installation, along the rail of which an ultrasonic sensor - range finder and a laser interferometer were fixed.

\section{Keywords}

Sensor, Ultrasonic Rangefinder, Laser interferometer, Measuring installation, Sensor calibration.

\section{Introduction and Problem Statement}

Ultrasonic sensors are often used as range finders [1]. Their main disadvantage is the lack of measurement accuracy, as it is due to the negative impact of the environment in which the sound propagates. Parameters and values of characteristics (the main of which is the optical density) can't be constant and can change during measurements.

An important disadvantage is the limitation of the measured distance to values from $3 \mathrm{~cm}$ to $40 \mathrm{~cm}$. It should also be noted the following disadvantages of ultrasonic sensors. The surfaces, which are characterized by a porous structure, absorb ultrasound well; therefore, it is difficult to measure the distance to them. If the distance to a surface at an angle to the beam or a spherical surface is to be measured, the obtained results may be inaccurate. The same problems await us when measuring the distance to the wall covered with foam. As result, the errors of such sensors range finders are accessed as $4 \mathrm{~cm}$ for the measured distance $20 \mathrm{~cm}-1400 \mathrm{~cm} \mathrm{[2]} \mathrm{error} 20 \%$ for $20 \mathrm{~cm}$ ) that seems to be insufficient for robot application.

These shortcomings require experimenters not only to take into account the above parameters while applying the ultrasonic sensors - range finders but to ensure the metrological unity of measurements with their help as well as to prove the exactness of the studied sensors. The latter can be achieved by developing metrological equipment for calibration of ultrasonic sensors - range finders.

\section{The Aim of the Work}

The purpose of the work is to study ultrasonic sensors and calibrate them with the help of a specially designed measuring installation based on a high-precision laser interferometer.

\section{Ultrasonic Sensor Calibration}

\subsection{Initial Data for Studies}

The ultrasonic distance sensor UGT593 (Fig. 1) of IFM Company, Germany, production has to be tested with the help of a specially designed measuring installation based on a laser interferometer MI 60 from SIOS, Germany [3].

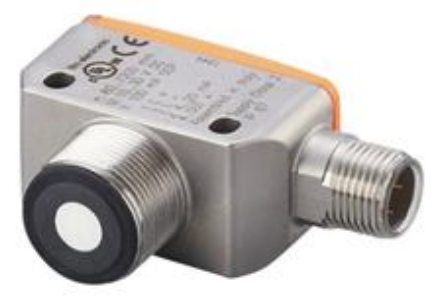

Fig.1. The ultrasonic sensor-rangefinder of the UGT593 type, IFM, Germany

Optical interferometers are considered as quite common instruments for measuring the wavelengths of spectral lines, refractive indices of transparent media, absolute and relative lengths, and angular sizes of stars, for controlling the quality of optical units and their surfaces, state of the surfaces, and so on. The principles of interferometers are the same; they differ only in the methods of obtaining and measuring coherent waves. The operation of interferometers is based on the spatial separation of the light bundle by a device into two or more coherent rays, which pass through 
different optical paths. Then they are brought together observing their interference result. The type of interference depends on the method of dividing the light bundle into mutually coherent rays, their number and relative intensity, the size of the source, and the light spectrum. Methods for obtaining coherent beams in interferometry are diverse. Therefore, there are a significant number of interferometer designs. According to the number of light beams, optical interferometers can be divided into multibeam and two-beam. Multibeam interferometers are used mainly as highresolution spectrometers to study the fine structure of spectral lines and determine their shapes; two-beam interferometers are mainly technical devices.

Initially, the readings of the ultrasonic sensor were investigated by self-analysis. The sensor was placed on a millimeter-scale (Fig. 2) to which the reference screen was attached. The characteristics of the sensor indicate that the measuring range is $60 \mathrm{~mm}-800 \mathrm{~mm}$, and the initial values - in $\mathrm{mA}$, from 4 to 20 . In previous studies, the sensor was installed at a distance from $70 \mathrm{~mm}$ to $400 \mathrm{~mm}$. The linear ratio of the device readouts in $\mathrm{mA}$ to $\mathrm{mm}$ was calculated. The obtained value of the output current $4 \mathrm{~mA}$ has corresponded to a distance of $70 \mathrm{~mm}$, and $20 \mathrm{~mA}$ corresponding to $400 \mathrm{~mm}$.

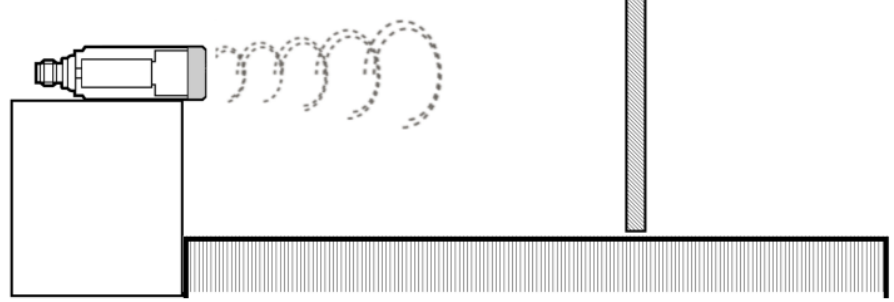

Fig.2. Scheme of ultrasonic sensor characteristics study

The next step in testing the sensor consisted of the application of a standard rail for providing the reflector movement. To direct the ultrasonic sensor's irradiation to the rear wall of the reflector, the irradiation area was enlarged by attaching a partition of $100 \times 100 \mathrm{~mm}^{2}$ to this wall (Fig. 3). The light bunch from a laser interferometer was directed to the front wall of the same reflector.

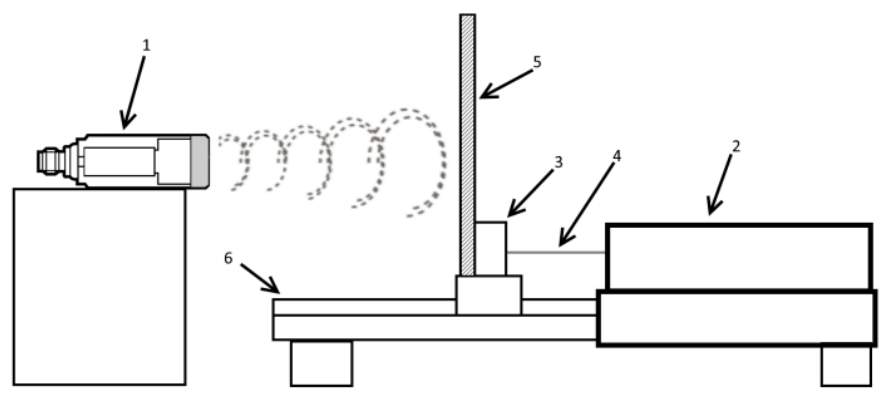

Fig.3. Scheme of the ultrasonic sensor studies installed on an optical bench: 1 is the ultrasonic sensor; 2 is an interferometer; 3 is the reflector; 4 is the light bunch; 5 is the partition; 6 - optical bench rail.

The sensor and interferometer were connected to a computer and have recorded their readouts for 6 minutes. Every one and a half minutes the reflector was shifted to the maximum possible distance of $21 \mathrm{~mm}$. The recorded data of the interferometer are shown in Fig.4, and the ultrasonic sensor - in Fig.5.

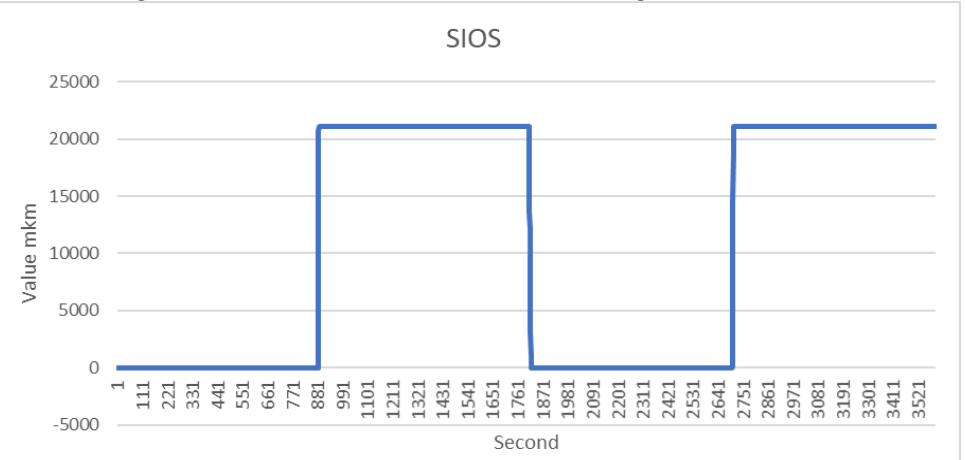

Fig.4. Interferometer data and their change during partition displacement 


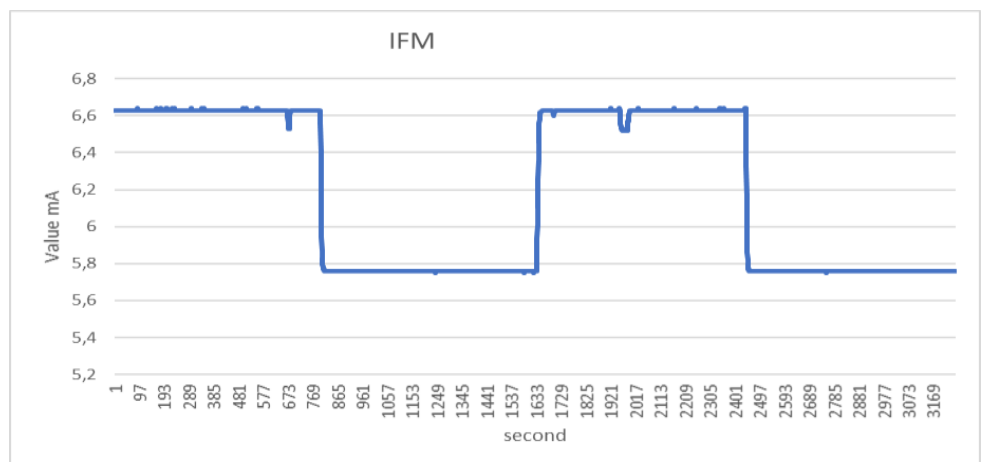

Fig.5. Ultrasonic sensor data and their change during partition displacement

\subsection{Calibration Background}

A) the table and optical bench vibration checking.

Before starting the measurement with the help of a laser interferometer, the table and the optical bench have been checked for vibrations. The measuring installation for this purpose is shown in Fig.6.

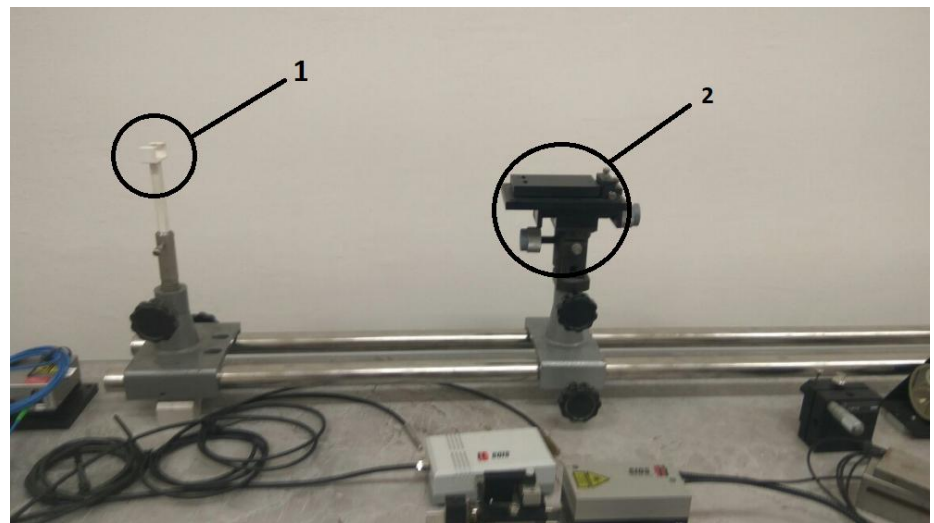

Fig.6. The installation for studies: 1 is the mounting of the mirror, 2 is the mounting of the interferometer.

The installation was checked as follows: the mirror was placed at a distance of $\sim 20 \mathrm{~cm}$, the interferometer was turned on. During the program start, the distance between the mirror and the interferometer was reset by the program to zero; then have been recorded the readings of the interferometer, due solely to the action of vibration impact on the installation (Fig. 7).

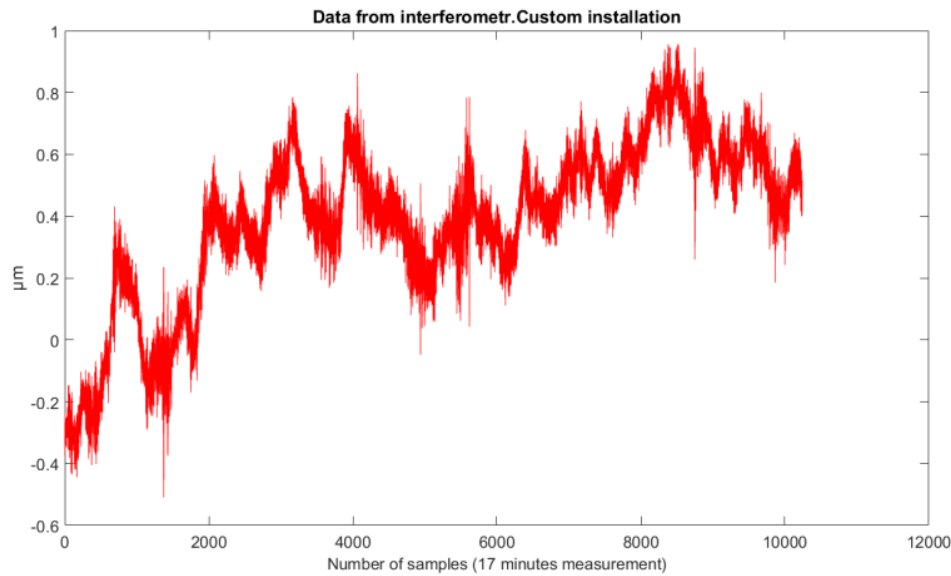

Fig. 7. Vibrations of the measuring installation, recorded by a laser interferometer (initial state).

While analyzing the graph, preliminary concluded that the obtained data may be incorrect, due to affecting the method of mounting a reflector mirror, inherent in its oscillations. To eliminate this effect, there was elaborated the mounting unit of the laser interferometer, SIOS, Germany, by inserting the bearing into it (Fig. 8).

Then, there were repeated measurements, the results of which are shown in Fig. 9. The amplitude of oscillations has decreased to the tens of nanometers. Such intensity is not able to affect the checking procedure for the ultrasonic sensor since its sensitivity is a priori at the level of $10^{5}$ nanometers. 


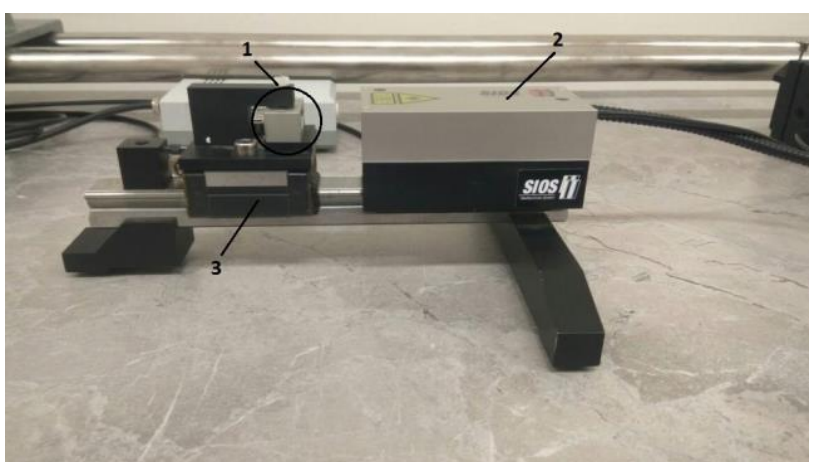

Fig. 8. Implementation of the laser interferometer: 1 is the reflective mirror, 2 is the laser interferometer, 3 is the moving unit mounted on the optical bench rail.

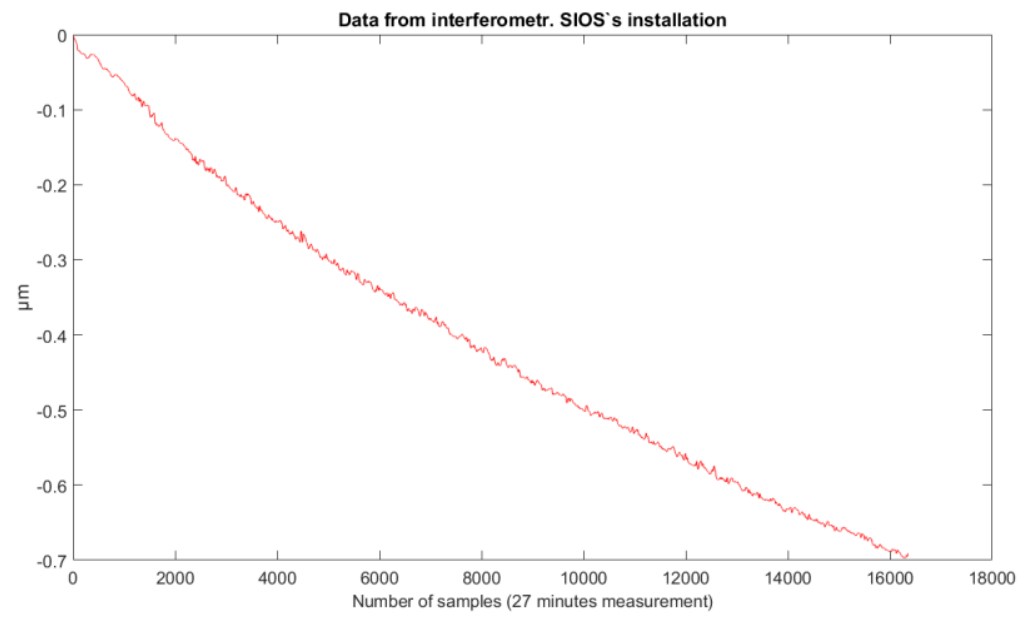

Fig.9. Vibration oscillations of the improved measuring installation fixed by the laser interferometer.

B) The identified following problem consists of the drift of the laser interferometer readings.

- First of all, the possible influence of an optical bench was tested. There was studied the possible impact of a bench table inclination. It could be caused by the drift of the installed bearing on which the mirror was fixed. The measuring device was turned $180^{\circ}$ and the bearing was mounted in the extreme position in the direction of the possible curvature of the table. Then, the bearing rested on the limiting edge of the installation and, as a result, immobilized aiming the elimination the readings drift. Such the test was performed twice (Fig. 10 a, b). The results of the measurements have demonstrated that the drift was not caused by the curvature of the table.

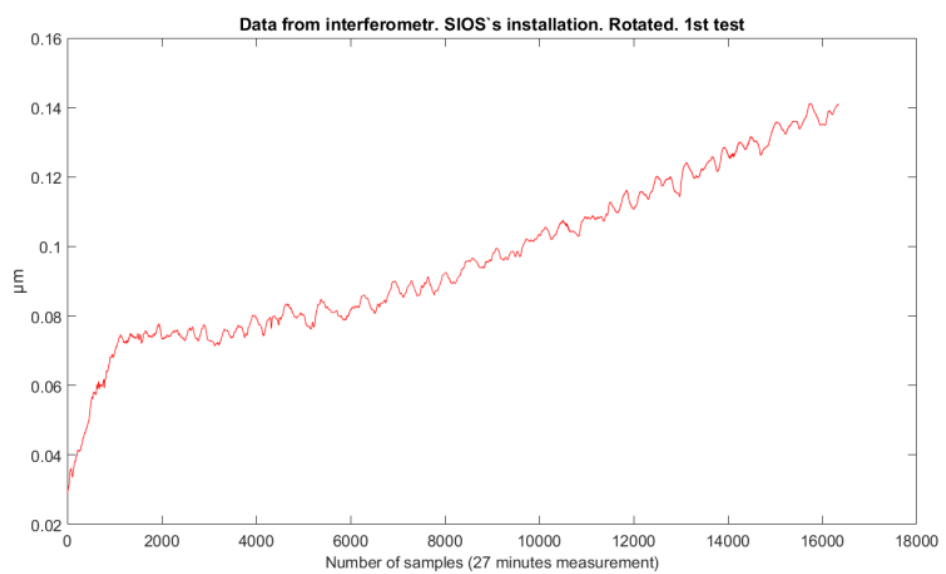

a) 


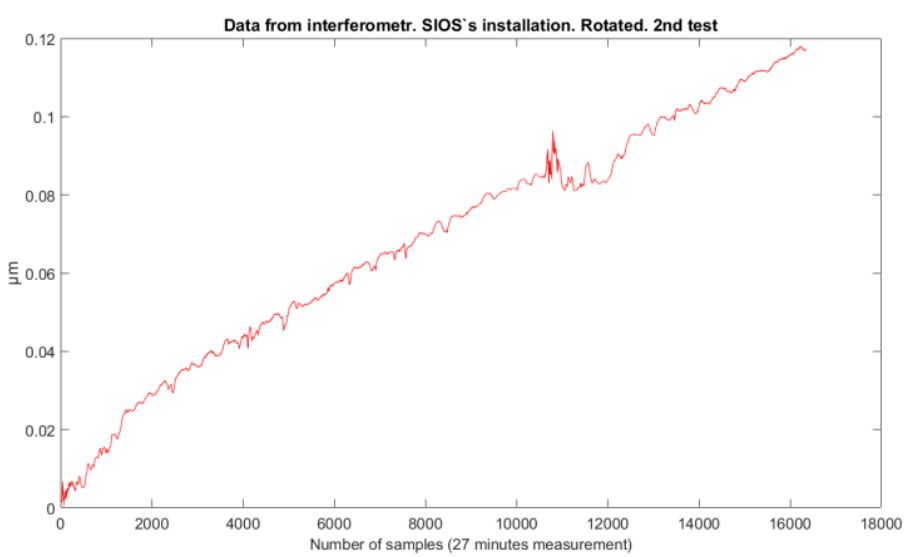

b)

Fig. 10. The measurement results for the rot4ated $180^{\circ}$ installation.

- Next, the nature of drift depending on the measurement distance to the interferometer was studied. To realize this, the measurement was performed while the reflective mirror was placed in the middle of the rail of the optical bench (Fig. 11, a). Then the bearing with the mirror was shifted severally to the left side (Fig. 11, b) and the right side (Fig. $11, \mathrm{c})$, and the measurements have been fulfilled.

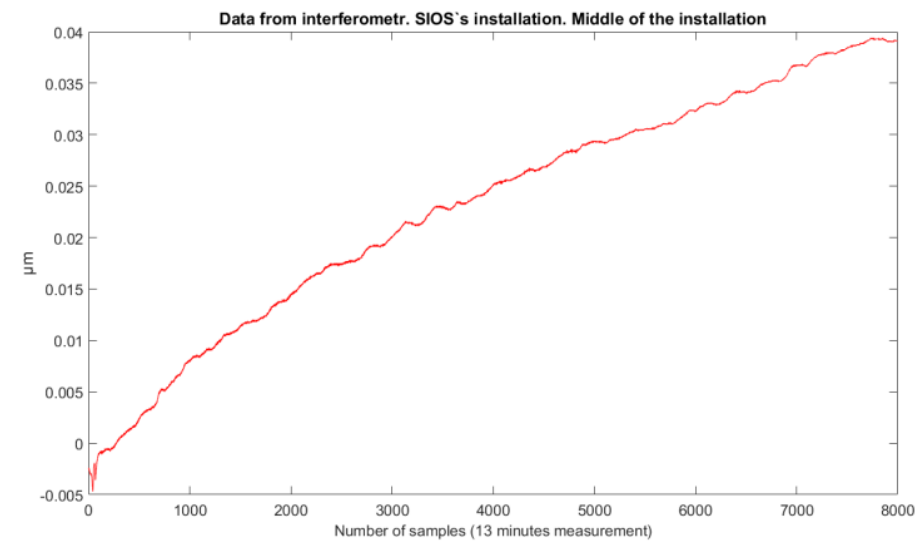

Fig. 11, a. The measurement results when placing the mirror in the central area of the rail of the optical bench

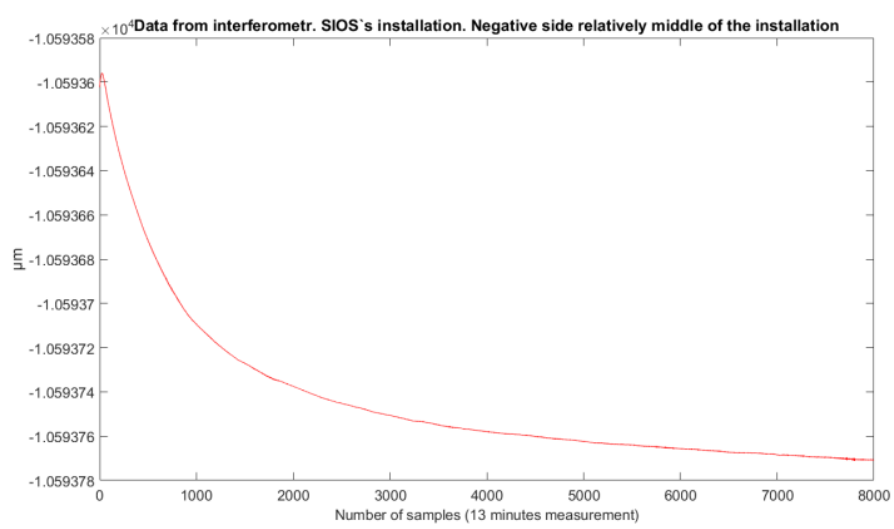

b) 


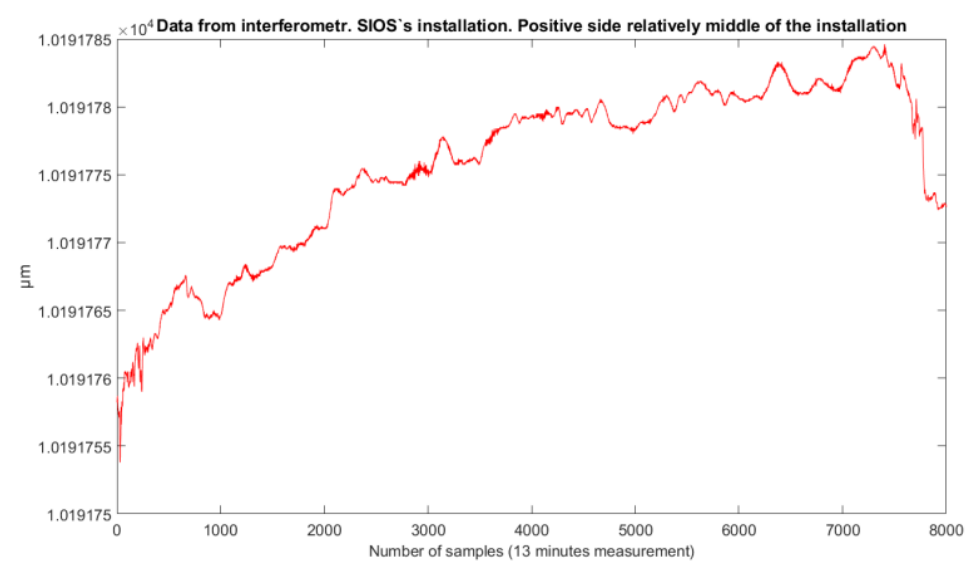

c)

Fig. 11, b, c. The measurement results when the bearing with the mirror was shifted severally to the left side (b) and the right side (c).

The stability of interferometer readings over time has been studied. A four-hour test was performed to study the drift of interferometer readings (Fig. 12). As can be seen, drift is stable over time. During the four-hour measurement, changes in environmental conditions (temperature, pressure, humidity, wavelength correction) were recorded.

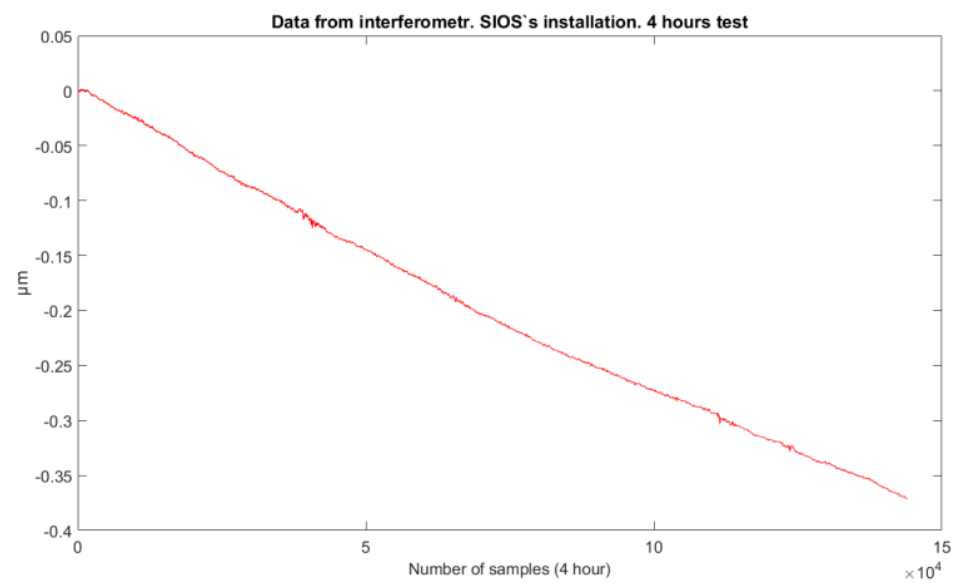

Fig. 12. Four-hour testing of the interferometer drift

The correlation between the drift of readouts and changes in environmental conditions has been studied that is the relationship between temperature and wavelength of the interferometer was controlled simultaneously (Fig. 13). The results have shown that the change in temperature affects the wavelength of light generated by the interferometer laser: the effect is directly proportional. However, the change in wavelength determines the changes in the readings of the interferometer, i.e. the reason for the drift of the reference measuring instrument while testing seems to be the temperature change. 


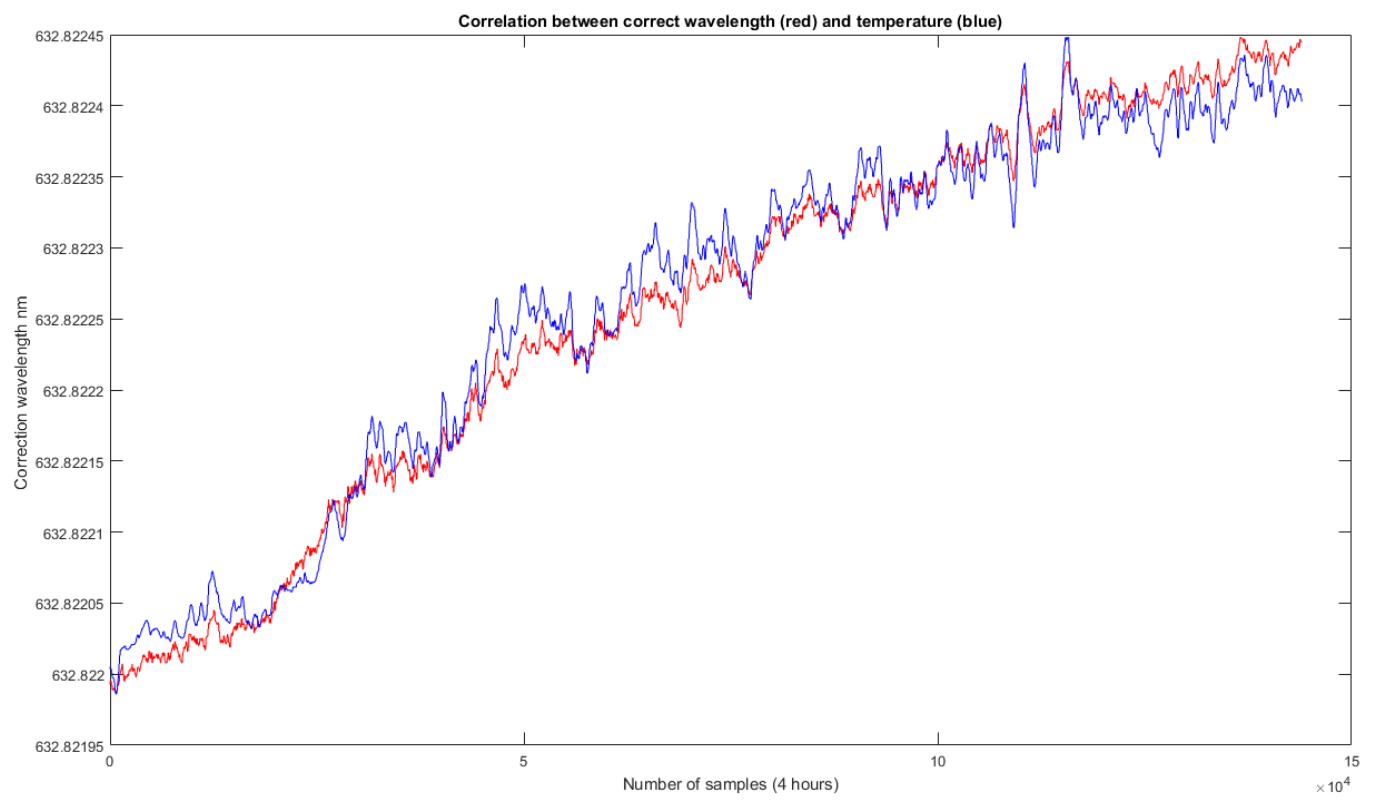

Fig.13. Checking of environment impact on the measurement results (blue color - the temperature at each moment, red color - the wavelength of the interferometer, controlled simultaneously).

Since the previously estimated change in the wavelength of light generated by the laser interferometer has demonstrated its insignificance - the changes were $0.00003 \%$ - further measurements were made (at the maximum possible distance), without stabilizing the temperature of the installation in general and the laser in particular. The results of testing the installation are shown in Fig.14. Here, the temperature does not the cause of the drift, estimated in time as $0,000007\left(7+10^{-6}\right)+10^{-4}=10^{-10} \mu \mathrm{m}=10^{-7} \mathrm{~nm}$ in 3.5 hours.

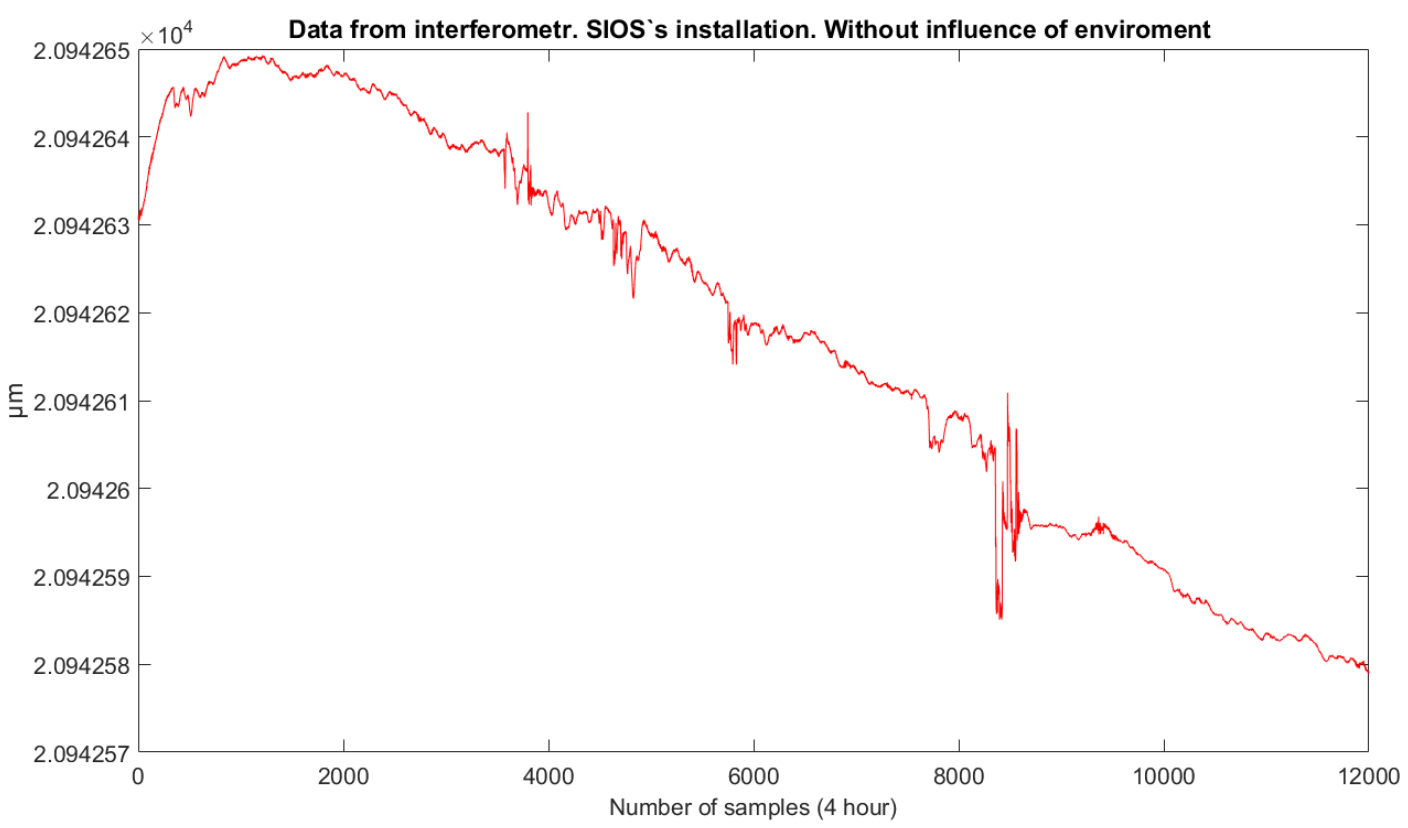

Fig. 14. Study of changes in the readings (drift) of the designed installation while thermostatic lack.

As a result of all kinds of tests, the drift of the interferometer readings, as the reference measuring instrument, did not exceed $1 \mu \mathrm{M}$. That is, the preparation of the designed installation based on a laser interferometer as a device with a basic reduced error $-1 \mu \mathrm{m}$ and the row of the additional errors error not exceeding $1 \mu \mathrm{m}$, confirmed the possibility of its application for calibrating the ultrasonic sensor-rangefinder type UGT593 of sensitivity 1000 $\mu \mathrm{m}$ [13]. 


\section{3. Calibration and Processing of Results}

Applying the high-mentioned installation, the calibration of the ultrasonic sensor UGT593 utilizing the laser interferometer. The results of the studies, repeated 5 times, are listed in Table 1.

Table 1. The results of calibration of the ultrasonic sensor by the laser interferometer

\begin{tabular}{|c|c|c|c|c|}
\hline $\begin{array}{c}\text { Number of the } \\
\text { calibration }\end{array}$ & \multicolumn{2}{|c|}{$\begin{array}{c}\text { Position of the reflective mirror in the } \\
\text { middle of the rail of the optical bench }\end{array}$} & $\begin{array}{c}\text { Displacement of the reflective mirror from the } \\
\text { middle of the rail of the optical bench }\end{array}$ \\
\cline { 2 - 5 } & $\begin{array}{c}\text { Sensor reading } I_{i z}, \\
m A\end{array}$ & $\begin{array}{c}\text { Interferometer } \\
\text { reading } l_{s z}, m m\end{array}$ & $\begin{array}{c}\text { Sensor readings } \\
I_{i d}, m A\end{array}$ & $\begin{array}{c}\text { Interferometer reading } \\
l_{s d}, m m\end{array}$ \\
\hline 1 & 6.63 & 0.0000002 & 5.76 & 21.0663187 \\
\hline 2 & 6.63 & 0.0000001 & 5.76 & 21.0663163 \\
\hline 3 & 6.63 & 0.0000003 & 5.76 & 21.0663137 \\
\hline 4 & 6.63 & 0.0000001 & 5.76 & 21.0663109 \\
\hline 5 & 6.63 & 0.0000001 & 5.76 & 21.0663085 \\
\hline
\end{tabular}

The received readings of the ultrasonic sensor in $\mathrm{mA}$ are converted into $\mathrm{mm}$ by using a linear relationship: $l_{i z}$, $\mathrm{mm}=$ $70+\left(\left(I_{i z}, \mathrm{~mA}-4\right)+400\right) /(20-4)($ Table 2$)$.

Table 2. The processed results of calibration of the ultrasonic sensor by the laser interferometer

\begin{tabular}{|c|c|c|c|c|c|c|}
\hline № & \multicolumn{2}{|c|}{$\begin{array}{c}\text { Position of the reflective } \\
\text { mirror in the middle of the } \\
\text { rail of the optical bench, mm }\end{array}$} & \multicolumn{3}{|c|}{ Displacement of the reflective mirror from the middle of the rail of the } \\
optical bench, mm
\end{tabular}

The processing of the obtained results, in particular the $5^{\text {th }}$ and $6^{\text {th }}$ columns of Table 2 , is given below. First of all, at one point of the optical bench (for the same location of the mirror) we find the average value of the interferometer $l_{s}$ $=21.06631346 \mathrm{~mm}$ and the ultrasonic sensor $l_{i}=21.75 \mathrm{~mm}$. Next, determine the calibration error of the ultrasonic sensor at the high-mentioned point of the optical bench: $\Delta l=l_{i}-l_{s}=0.6836 \mathrm{~mm}$; when the variance of the average readings of the interferometer $S=1.65556+10^{-11} \mathrm{~mm}$; the standard deviation of interferometer readings $S_{I}=$ $4.06886+10^{-6} \mathrm{~mm}$; the uncertainty of interferometer readings $U=1.81965+10-6 \mathrm{~mm}$ provided by five repetitions of measurements.

\section{Conclusions}

The metrological installation for calibration of the ultrasonic sensor-rangefinder UGT593, IFM, Germany utilizing the laser interferometer, SIOS, Germany is offered and its realization based on an optical bench. Some factors influencing the readouts of the latter have been previously studied. This made it possible to eliminate several systematic errors, in particular, due to the imperfection of the installation and fixing of the interferometer; the movement of the reflector, concerning which the calibration has been performed simultaneously according to the sensor and the interferometer readings, as well as errors caused by environmental impacts, including temperature drift (the latter, as shown, can be neglected). The sensor itself was calibrated five times and its metrological parameters were determined, including the error of distance measurement by it. The error of distance measurement determined by us with the help of the studied sensor was $0.6836 \mathrm{~mm}$ at a distance of $\sim 21 \mathrm{~mm}$ or $3,25 \%$. 


\section{Acknowledgments}

The authors express their gratitude to the staff of the Department of Information-Measuring Technologies of Lviv Polytechnic National University, Ukraine, for the assistance in the preparation of this article.

\section{Conflict of Interests}

Conflict of interest while writing, preparing, and publishing the article as well as mutual claims by the co-authors is absent.

\section{References}

[1] Yo. Na, "The design of ultrasonic range finder", in Proc. AIP Conf. Proc., 1820, 060024 Publ. Online: 13 March 2017. https://doi.org/10.1063/1.4977339

[2] V. Mazzari, "Ultrasound sensor. High quality ultrasound sensors", Robotics tutorials, tagged Hokuyo, lego mindstorms, SICK, TeraRanger, TOF, Ultrasound sensor on 21March, 2017. https://blog.generationrobots.com/en/ultrasound-sensor-high-qualityultrasound-sensors-available-now/

[3] E. Manske, G. Jager, R. Mastylo, D. Dontsov, "Nanopositioning abd Nanomeasuring Machine for Multi-Sensor Application", Measuring Equipment and Metrology, vol.81, no.1, pp.17-24, 2020. 